\title{
USO DE MARCADORES SSR PARA IDENTIFICACIÓN \\ DE GERMOPLASMA DE PAPA EN EL PROGRAMA DE MEJORAMIENTO DE INIA DE CHILE
}

\section{Use of SSR markers to identify potato germplasm in the INIA Chile breeding program}

\author{
Mónica Mathias R. ${ }^{1}$, Boris Sagredo D. ${ }^{2}$, Julio Kalazich B. ${ }^{2}$
}

\begin{abstract}
A B S T R A C T
Molecular markers based on Simple Sequence Repeats (SSR) are a very efficient tool for potato (Solanum tuberosum L.) genotype identification and can be very useful for germplasm conservation and management. With the purpose of incorporate this technology into the potato breeding program of the National Institute of Agricultural Research (INIA) Chile, a set of 26 SSR markers was evaluated on a sample of 71 potato genotypes. Each marker was characterized for number and combinations of alleles, scoring quality, polymorphic information content (PIC) and discrimination power (D). From the total, only 21 SSR markers showed up scoreable products and the allele number ranged between 2 and 17. The observed allelic combinations among the different potato genotypes ranged from 2 to 47; however, unique genotypes detected by each SSR marker ranged from 0 to 38. The observed (Do) and expected $(\mathrm{Dj})$ discriminatory power ranged from 0.23 to 0.98 and from 0.43 to 0.92 , respectively. The seven SSR markers which showed the highest Do scores were STM1009 (0.98), STM1020 (0.97), STM0031 (0.97), STM2013 (0.96), STM 1008 (0.94), STM1052 (0.93) and STM0019 (0.91). The STM1009, STM1020 and STM1008 markers are multi-loci SSR, where each one amplifies more than one locus of the potato genome. The utilization of the multi-loci type of marker, or combinations of several SSR markers in either PCR-multiplex or pseudo-multiplex reactions, are good options to increase the speed and reduce the cost of SSR markers application.
\end{abstract}

Key words: Solanum tuberosum, microsatellite, DNAfingerprint.

\section{R E S U M E N}

Los marcadores moleculares basados en Secuencias Simples Repetidas (SSR) constituyen una herramienta altamente eficaz para la identificación de genotipos de papa (Solanum tuberosum L.) y pueden ser de gran utilidad en la conservación y manejo de germoplasma. Con el propósito de incorporar esta tecnología al Programa de Mejoramiento de Papa del Instituto de Investigaciones Agropecuarias (INIA) de Chile, se evaluó un grupo de 26 marcadores SSR sobre una muestra de 71 genotipos de papa. Cada marcador se caracterizó según su número de alelos y sus respectivas combinaciones, calidad de lectura, contenido de información polimórfica (PIC) y poder discriminatorio (D). Del total sólo 21 marcadores SSR mostraron productos legibles con un número de alelos que varió entre 2 y 17. Las combinaciones alélicas observadas variaron desde 2 a 47; sin embargo, los genotipos únicos detectados por cada marcador fueron desde 0 a 38. El poder discriminatorio observado (Do) y esperado (Dj) estuvo entre 0,23 a 0,98 y entre 0,43 a 0,92 , respectivamente. Los siete marcadores que presentaron mayor Do fueron STM1009 $(0,98)$, STM1020 $(0,97)$, STM0031 (0,97), STM2013 (0,96), STM1 008 $(0,94)$, STM1 $052(0,93)$ y STM0019 $(0,91)$. Los marcadores STM1009, STM1020 y STM1008 corresponden a SSR multi-loci, donde cada uno amplifica más de un locus desde distintas regiones del genoma de la papa. La utilización de este tipo de marcadores multi-loci, o de combinaciones de varios SSR en reacciones de PCR-múltiplex o pseudos-múltiplex son una buena alternativa para aumentar rapidez y disminuir costo en la aplicación de marcadores SSR.

Palabras clave: Solanum tuberosum, microsatélites, huella digital de ADN.

\footnotetext{
${ }^{1}$ Instituto de Investigaciones Agropecuarias, Centro Regional de Investigación Carillanca, Casilla 58-D, Temuco, Chile.

${ }^{2}$ Instituto de Investigaciones Agropecuarias, Centro Regional de Investigación Remehue, Casilla 24-D, Osorno, Chile.

E-mail: bsagredo@inia.c *Autor para correspondencia.

Recibido: 15 de marzo de 2006. Aceptado: 18 de julio de 2006.
} 


\section{INTRODUCCIÓN}

Los programas de mejoramiento genético de papa (Solanum tuberosum L.) necesitan conservar y manejar colecciones de germoplasma, conformadas por numerosos genotipos que son utilizados como progenitores. La mayoría de estos genotipos son portadores de genes valiosos, que a través de cruzamientos son transferidos a los futuros nuevos cultivares.

Actualmente el germoplasma de papa del Programa de Mejoramiento Genético de Papa del Instituto de Investigaciones Agropecuarias (INIA), del Centro Regional de Investigación Remehue (CRI), está constituido por aproximadamente 500 genotipos que son mantenidos bajo condiciones de cultivo in vitro y en campo. El tamaño de esta colección está en constante crecimiento y regularmente se integran nuevos genotipos, los cuales provienen de las selecciones avanzadas del mismo programa y de introducciones de materiales de otros programas y regiones.

La mantención del germoplasma de INIA bajo condiciones de cultivo in vitro requiere que cada genotipo sea repicado a lo menos dos a tres veces al año, siendo indispensable contar con un sistema de etiquetado e identificación confiable que evite confusiones, que podrían generar mezclas que pueden llevar a pérdidas de materiales valiosos. En condiciones de campo también se pueden producir este tipo de confusiones, especialmente en un programa de mejoramiento donde se realizan cientos de cruzamientos que generan más de 50.000 genotipos nuevos cada temporada.

En el caso de los cultivares descritos oficialmente, éstos podrían ser identificados a través de descriptores morfo-agronómicos como los descritos por la Unión Internacional para la Protección de las Obtenciones Vegetales (UPOV, 2001). Sin embargo, esta metodología requiere de al menos una temporada completa de cultivo, y puede ser afectada por el medio ambiente, por lo que en casos de confusión sea más conveniente readquirir el cultivar desde una fuente confiable. Más dramático es el caso de los clones que se liberan como germoplasma, que normalmente no son caracterizados suficientemente, y sus descriptores morfoagronómicos suelen ser limitados para su correcta identificación.
La identificación molecular basada en pruebas de ADN-fingerprint utilizando marcadores de microsatélites (SSRs) ha sido descrita como una poderosa herramienta de identificación de clones y cultivares de papa que podría facilitar enormemente la conservación y manejo del germoplasma de papa (Perazzo et al., 2001; Ghislain et al., 2001a; b). El Laboratorio de Biología Molecular Aplicada del Centro Internacional de la Papa (CIP), ha utilizado este tipo de marcadores para evaluar el nivel de error en sus sistemas de etiquetado dentro de grandes colecciones de germoplasma que superan las 5.000 accesiones (Perazzo et al., 2001). La incorporación de este tipo de herramientas a nuestro programa de mejoramiento de papa facilitará el manejo de colecciones, tanto in vitro como en campo, y será de gran apoyo a las actividades de intercambio de germoplasma con los distintos centros y programas dedicados a esta actividad.

Los marcadores moleculares corresponden a regiones del ADN que muestran polimorfismo o variación entre diferentes individuos dentro de una especie (Liu, 1998). El análisis de ADN presenta múltiples ventajas, por entregar información genética precisa, permitir el análisis simultáneo de un gran número de muestras y es independiente del medio ambiente. Por otro lado, el ADN puede ser extraído a partir de cantidades ínfimas de tejido de cualquier órgano somático y estado de desarrollo de la planta, permitiendo la obtención de resultados en un corto plazo.

En Chile, el uso de técnicas moleculares para identificación de plantas es aún incipiente, observándose un mayor desarrollo en el área vitivinícola (Narvaez, 1998; Narvaez et al., 2001). Sin embargo, existen algunos trabajos de caracterización molecular realizados en arroz (Hinrichsen et al., 1996), trigo (Zerené et al., 2000), frutillas (Becerra et al., 2001), accesiones silvestres de papa (Riegel et al., 2004) y material genético forestal (Paredes et al., 2004).

Se han descrito diversos tipos de marcadores de ADN útiles para la identificación de genotipos y cultivares de papa tales como RFLP, AFLP, RAPD y SSR (Ortiz et al., 2000). Sin embargo, los más utilizados y aceptados han sido los SSRs (Single Sequence Repeat) o microsatélites (Schneider y Douches, 1997; Ashkenazi et al., 2001; Ghislain et al., 2001b; 2004; Norero et al., 2003). Los SSRs 
presentan mayor simplicidad tecnológica en relación a RFLP y AFLP, y no requieren alta concentración y calidad de ADN. Por otro lado, los SSRs al contrario de los marcadores RAPD, presentan alta reproducibilidad entre laboratorios y son de base genética codominante, y generalmente permiten detectar todos los alelos posibles de un locus (Ortiz et al., 2000).

Los microsatélites se encuentran ampliamente distribuidos en los genomas de organismos eucariotas, y a nivel molecular corresponden a secuencias de 1 a 5 nucleótidos repetidas en tándem, en donde el número de repeticiones revela diferencias genéticas entre individuos (Kruglyak et al., 1998). En general, el grado de polimorfismo revelado aumenta con la longitud total del SSR, y aunque un microsatélite no es específico de un locus, las regiones flanqueantes sí lo son, por lo que un par de partidores para estas regiones amplificará un microsatélite concreto (Ortiz et al., 2000).

El ADN conteniendo secuencias tipo microsatélites puede ser amplificado usando la Reacción en Cadena de la Polimerasa (PCR), con partidores que flanquean el motivo repetitivo y pueden ser fácilmente resueltos por electroforesis (Liu, 1998). Los patrones de fragmentos de ADN observados por tinción con sales de plata corresponden a una huella digital, o fingerprint, genético distintivo de cada cultivar. Existen otros sistemas alternativos de visualización de los fragmentos de ADN que se basan en el uso de isótopos radiactivos o fluoróforos (Sambrook y Russell, 2001). En papa la tecnología SSR ha sido desarrollada usando ADN de cultivares modernos (Ghislain et al., 2004). La primera generación de SSR fue obtenida a partir de la identificación de motivos repetitivos en secuencias de genes (Veilleux et al., 1995; Kawchuck et al., 1996; Provan et al., 1996; Schneider y Douches, 1997). El segundo grupo de SSRs surgió del análisis de librerías genómicas enriquecidas con motivos repetitivos (Milbourne et al., 1998). Más recientemente, se ha reportado la búsqueda de motivos repetitivos dentro de las bases de datos de EST (Expressed Sequence Tag) y librerías de cDNA provenientes de papa, donde el $5 \%$ de ellos contienen SSR (Ghislain et al., 2004, Feingold et al., 2005).

En este artículo se presentan los resultados de la evaluación de 26 marcadores SSR sobre un grupo de 71 genotipos de papa pertenecientes al germo- plasma del Programa de Mejoramiento de INIA, Chile. Además, se proponen alternativas para reducir costos y tiempo de análisis, que incluyen modificaciones a los procesos de extracción de ADN y la utilización de marcadores SSR en reacciones de multi-loci, múltiplex y pseudos-múltiplex.

\section{MATERIALES Y MÉTODOS}

\section{Material vegetal}

Se estudió un grupo de 71 genotipos de papa correspondientes a 35 cultivares comerciales, 12 clones avanzados del Programa de Mejoramiento de INIA, Chile, nueve clones originarios del programa de mejoramiento de la Universidad de Cornell, EE.UU., y 15 clones provenientes del Centro Internacional de la Papa (CIP), Lima, Perú. Este material es mantenido in vitro y en campo, en el Centro Regional de Investigación Remehue del INIA, Osorno, Chile.

\section{Extracción de ADN}

El ADN de cada genotipo se obtuvo a partir de hojas frescas provenientes de plántulas mantenidas in vitro o de plantas crecidas en invernadero, utilizando la metodología descrita por Fulton et al. (1995). Además, con el objetivo de introducir un método de extracción de ADN más sencillo y rápido que el anterior, se evaluó el protocolo descrito por Paris y Carter (2000) con leves modificaciones, lo que consistió en la molienda de un disco de hoja en $\mathrm{NaOH}$ $250 \mathrm{mM}$ y posteriormente, la transferencia del sobrenadante en seis volúmenes de solución concentrada de TE (Tris-HCl $80 \mathrm{mM} \mathrm{pH} \mathrm{7,5} \mathrm{y} \mathrm{EDTA} 1$ M). El ADN contenido en esta solución resultante se utilizó directamente como templado en las reacciones de PCR.

\section{Loci SSRs y condiciones de amplificación por PCR}

Se utilizó un grupo de 26 marcadores microsatélites descritos por Milbourne et al. (1998) (Cuadros $1 \mathrm{y}$ 2). Todos los marcadores SSRs se evaluaron individualmente, excepto el par STM3012/STPoAc58 que se evaluó sólo como un múltiplex, que consiste en realizar una reacción de PCR simultánea para más de un SSR. Además, algunos marcadores se analizaron como pseudos-múltiplex (STM1052/ STM2013 y STM1016/STM1104/STGBSS), que consiste en mezclar los productos PCR de SSRs que presentan productos de diferente tamaño (Ghislain 


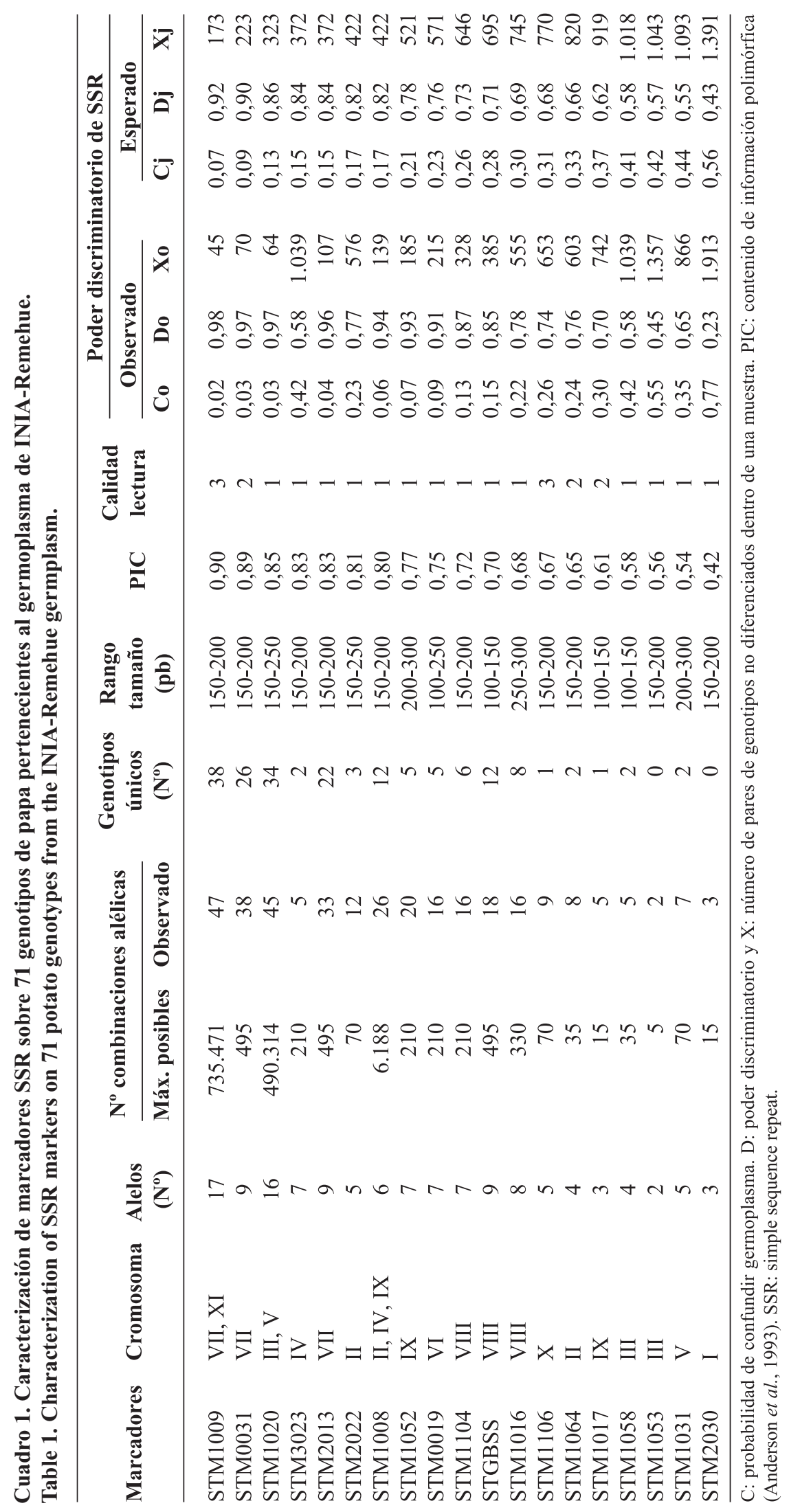




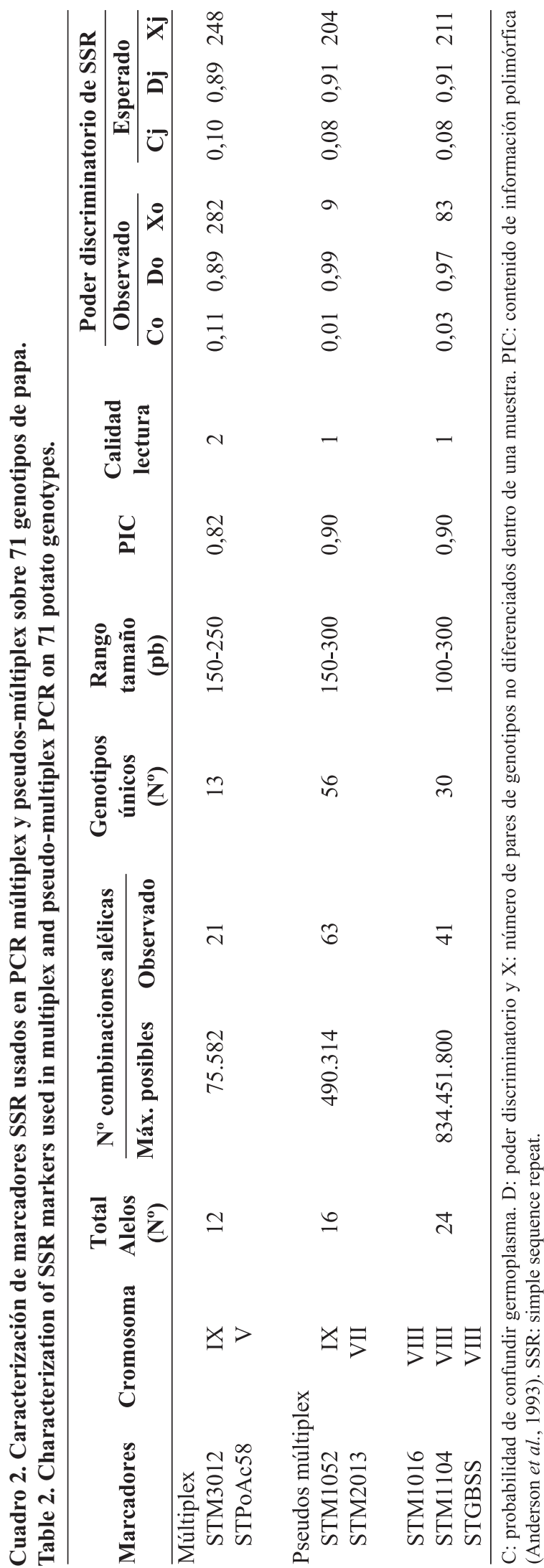

et al., 2004). En todos los casos la mezcla de PCR consistió en $20 \mu \mathrm{L}$ de volumen final con aproximadamente 10-20 ng de ADN templado, $1 \mathrm{X}$ de buffer PCR (Tris $20 \mathrm{mM}, \mathrm{KCl} 50 \mathrm{mM}, \mathrm{pH} 8,4$ ), 1,2 a 2,0 $\mathrm{mM}$ de $\mathrm{MgCl}_{2}, 0,125 \mathrm{mM}$ de cada dNTP, $5 \mu \mathrm{M}$ de cada partidor y $1 \mathrm{U}$ de enzima Taq polimerasa. El programa de PCR consistió en un ciclo de desnaturación a $94^{\circ} \mathrm{C}$ por $3 \mathrm{~min}$; 40 ciclos con desnaturación a $94^{\circ} \mathrm{C}$ por $30 \mathrm{~s}$, alineamiento de 50 a $60^{\circ} \mathrm{C}$ por 1 min dependiendo del partidor, y elongación a $72^{\circ} \mathrm{C}$ por $1 \mathrm{~min}$; finalmente un ciclo de extensión a $72^{\circ} \mathrm{C}$ por 5 min (Milbourne et al., 1998). Los fragmentos amplificados por PCR se separaron en geles de secuenciación de poliacrilamida al 6\% (20:1 acrilamida:bisacrilamida) con $7 \mathrm{M}$ urea. La electroforesis se realizó a 1.700 Volts por $2 \mathrm{~h}$ y finalmente las bandas se visualizaron a través de tinción con sales de plata (Promega, 2004).

\section{Lectura de alelos de SSR}

Cada alelo de SSR correspondiente a una banda amplificada se registró con “1” ó “ 0 ”, según su presencia o ausencia, respectivamente, para cada genotipo, y se ingresó en una matriz de datos. Las bandas tenues de apariencia diferente a la del patrón dominante se consideraron como artefactos de la PCR y no se incluyeron en el análisis. Cada SSR se caracterizó según la nitidez de sus bandas, ancho o amplitud de sus alelos y presencia de sombras, definiéndose un índice de calidad de lectura para cada marcador SSR de acuerdo a la metodología consensuada en el Taller de análisis SSR en papa y desarrollo de bases de datos, realizado en el Centro Internacional de la Papa (CIP, 2004) (Cuadros 3 y 4). El tamaño de los alelos se determinó por comparación de movilidad en el gel con el marcador de tamaño molecular Gene Ruler ${ }^{\mathrm{TM}} 50$ pb DNA Ladder (MBI Fermentas, Ontario, Canadá). Cada uno de los geles teñidos con sales de plata se escaneó y almacenó en una base de datos digital quedando disponible para posteriores análisis y para quien lo solicite al autor de correspondencia.

\section{Determinación del poder discriminatorio de los SSRs}

Para cada marcador se estimó el poder discriminatorio esperado $(\mathrm{Dj})$ y observado (Do). El valor de Dj se estimó utilizando la metodología de Tessier et al. (1999). Considerando que C es la probabilidad de confusión, la probabilidad de que dos individuos elegidos al azar tengan diferentes patrones corresponde a $\mathrm{D}=1$ - $\mathrm{C}$. En un set de $\mathrm{N}$ individuos 
Cuadro 3. Parámetros de calidad de lectura de bandas de ADN de marcadores SSRs. Table 3. Parameters of scoring quality of DNA band of SSRs markers.

\begin{tabular}{clc}
\hline Parámetros y tipos de banda & Característica del tipo de banda & Puntaje \\
\hline Nitidez de banda & & 3 \\
T1 & Bandas nítidas & 1 \\
T2 & Presencia de bandas tartamudas de similar tamaño & 0 \\
T3 & Bandas borrosas & \\
Amplitud de alelos & Amplitud menos a 4 mm & 2 \\
A & Amplitud igual o superior a 5 mm & 1 \\
B & & 2 \\
Perfil de banda & Ausencia de sombras & 1 \\
I & Presencia de sombras & 1 \\
II &
\end{tabular}

Fuente: CIP. 2004. Taller de análisis SSR en papa y desarrollo de bases de datos. SSR: Simple sequence repeat.

es posible sortear $\mathrm{N}(\mathrm{N}-1) / 2$ pares diferentes y si el $i$-ésimo patrón que entrega el partidor $j$-ésimo, presenta una frecuencia $\mathrm{p}_{i}$ dentro de este grupo de genotipos, la probabilidad de confusión $\mathrm{c}_{i}$ es:

$$
\mathrm{c}_{i}=\mathrm{p}_{i}\left(\mathrm{~N} \mathrm{p}_{i}-1\right) /(\mathrm{N}-1)
$$

El valor $\mathrm{C}_{\mathrm{j}}$ corresponde a la sumatoria de $\mathrm{c}_{i}$ de todos los patrones $/$ generados por el partidor. De esta forma el poder discriminatorio del partidor $j$-ésimo es igual a:

$$
\mathrm{D}_{\mathrm{j}}=1-\mathrm{C}_{\mathrm{j}}
$$

Como $\mathrm{N}$ tiende al infinito el límite de $\mathrm{D}_{j}$, igual a $\mathrm{D}_{\mathrm{L}}$, provee una estimación del poder discriminatorio de cada uno de los partidores $j$.

$$
\operatorname{Lim}\left(\mathrm{D}_{j}\right)=\mathrm{D}_{\mathrm{L}}=1-\sum \mathrm{p}_{i}^{2}
$$

Ésta corresponde a una extensión del Contenido de Información Polimórfica (PIC) (Anderson et

Cuadro 4. Estimación del índice de calidad de lectura de SSRs según sus parámetros de banda de ADN.

Table 4. Estimation of SSRs scoring quality index according their DNA band parameters.

\begin{tabular}{lcc}
\hline Clasificación de SSRs & $\begin{array}{c}\text { Puntaje } \\
\text { acumulado }\end{array}$ & $\begin{array}{c}\text { Índice } \\
\text { calidad lectura }\end{array}$ \\
\hline T1,A,I & 7 & 3 \\
T1,A,II - T1,B,I & 6 & 3 \\
T1,B,II - T2,A,I & 5 & 2 \\
T2,A,II - T2,B,I - T3,A,I & 4 & 2 \\
T2,B,I - T3,A,II - T3,B,I & 3 & 1 \\
T3,B,II & 2 & 1 \\
\hline
\end{tabular}

SSR: Simple sequence repeat. al., 1993) disponible desde las frecuencias de los diferentes patrones de bandas generados por un partidor.

El poder discriminatorio observado se estimó como Do $=1$ - Co, donde Co corresponde al porcentaje de la suma de pares de grupos de genotipos que presentaron un patrón de alelos SSR idénticos. Este valor se obtuvo de la siguiente manera: i) para cada marcador SSR se identificaron los grupos de genotipos idénticos, ii) luego separadamente se calculó el número de pares posibles que se forman dentro de cada grupo de genotipos idénticos, lo que corresponde al número de pares de genotipos que pueden ser confundidos entre sí, y iii) el porcentaje de la sumatoria total de todos estos pares de genotipos representa la probabilidad de confusión observada $(\mathrm{Co})$. Esta tarea se facilitó a través del uso de dendrogramas, que se obtuvieron por análisis de distancias genéticas (simple matching coefficient) y agrupamiento (UPGMA), utilizando el software NTSYSpc versión 2.02c (Sneath y Sokal, 1973).

\section{Combinaciones óptimas de SSR}

Para establecer las mejores combinaciones de marcadores SSRs, que permitan diferenciar el germoplasma evaluado, se utilizó la metodología de Tessier et al. (1999). Se elaboró una lista de marcadores ordenándolos según su PIC en forma decreciente. Luego, al marcador de mayor PIC se combinó el marcador inmediatamente menor, y así sucesivamente. Entonces, se estimó la capacidad discriminatoria acumulada esperada (Dk) de las distintas combinaciones de marcadores y su correspondiente número total de pares de genotipos no diferenciados Xk. Los respectivos valores observados 
(Do y Xo) de las diferentes combinaciones de marcadores SSR, se obtuvieron por análisis de dendrogramas con una metodología similar a la utilizada para los marcadores individuales, descrita anteriormente.

\section{Combinaciones alélicas}

Para una especie autotetraploide como S. tuberosum el número máximo de combinaciones alélicas posibles (Aj) para cada marcador SSR, se dedujo de la ecuación general de la teoría combinatoria para agrupaciones tipo combinación, completamente al azar y con posibilidades de repetición.

$$
\mathrm{CR}_{m}^{n}=\left(\frac{m+n-1}{n}\right)=\frac{(m+n-1) !}{n !(m-1) !}=\mathrm{Aj}
$$

Donde $m$ corresponde al número total de elementos disponibles y $n$ representa al número de elementos por grupo que se forman. Así en el caso de una población de individuos tetraploides, $m$ corresponde al número total de alelos por locus o loci contabilizados en la población analizada y $n$ al número máximo de alelos que se podría encontrar en un solo individuo de esta misma población. El valor de $n$ variará en múltiplos de cuatro $n=4, n=8, n=12$, $n=16, \ldots$ y $n=4 l$ para $1,2,3,4, \ldots$ y $l$ loci, respectivamente, de acuerdo al número de loci considerado en el análisis, ya sea productos SSR de una reacción PCR de simple locus $(l=1)$, multi-loci $(l>1)$, múltiplex ( $l>1)$, pseudos-múltiplex $(l>1)$. Por lo tanto, el número máximo de combinaciones alélicas posibles (Aj) para 1, 2 y 3 loci de acuerdo al tipo de marcador utilizado en este estudio se calculó de acuerdo a las siguientes ecuaciones:

$\mathrm{Aj}=\frac{(m+3) !}{4 !(m-1) !}$ para 1 locus $; \mathrm{Aj}=\frac{(m+7) !}{8 !(m-1) !}$ para 2 loci $;$

$\mathrm{Aj}=\frac{(m+11) !}{12 !(m-1) !}$ para 3 loci

El número de combinaciones alélicas observadas (Ao) y el número de genotipos únicos se determinó mediante recuento de ramas totales y/o únicas en dendrogramas generados por análisis de similitud, según se describió anteriormente.

\section{RESULTADOS Y DISCUSIÓN}

En este trabajo se reportan los resultados de la evaluación de 26 marcadores SSR, sobre un grupo de 71 clones y cultivares de papa de diferente origen. Los marcadores SSR utilizados corresponden a par- te del grupo descrito por Milbourne et al. (1998) y para su selección se consideró una buena distribución entre los grupos de ligamiento de la papa. Algunos de estos SSRs han sido recomendados para la identificación de cultivares de papa (Ghislain et al., 2004). Además, en este grupo se incluyeron marcadores SSR multi-loci que amplifican productos a partir de más de un locus y que fueron mapeados en distintos cromosomas (Milbourne et al., 1998). En cuanto al germoplasma de papa utilizado, a partir de la colección de más de 500 genotipos que se maneja en el programa de mejoramiento genético de papa de INIA-Chile, se eligió un grupo que representa por un lado la máxima diversidad en cuanto a su origen, y por otro constituye el grupo de progenitores más frecuentemente utilizados en los cruzamientos que se realizan anualmente en las actividades de mejoramiento, incluyendo materiales chilenos, sudamericanos, norteamericanos y europeos.

En una primera etapa de evaluación se consideró exclusivamente la calidad visible de los marcadores SSR y se descartaron del análisis todos aquellos marcadores que, bajo nuestras condiciones experimentales de laboratorio, resultaron en productos imposibles de escrutar. Los marcadores eliminados correspondieron a STM1050, STM0040, STM0030, STM0037 y STWAX-2, siendo los últimos tres SSRs utilizados con éxito en otros laboratorios (Gishlain et al., 2004). Esta diferencia puede explicarse por los diferentes tipos de germoplasma evaluados, problemas técnicos como degradación de partidores, o simplemente por las condiciones distintas de trabajo entre laboratorios.

Los 21 marcadores restantes que generaron productos escrutables, se caracterizaron según la calidad. Para ello se consideró la nitidez, la presencia de sombras que confunden la lectura, bandas tartamudas o presencia de fragmentos-artefactos de similar tamaño que dificultan el reconocimiento del alelo; finalmente la mayor amplitud de alelo, medida como la separación en mm entre las dos hebras desnaturadas de un mismo alelo fue considerada como un factor negativo, ya que genera el traslapamiento entre alelos diferentes (Cuadro 3). Así se construyó una escala de puntajes asignados a cada característica, de forma que los valores promedios fluctúan entre 1 y 3 , siendo los SSRs de mayor calidad los de tipo 1 (Cuadro 4). En la Figura 1 se muestran los marcadores SSR STM1053, STM0031 y STM1009 que obtuvieron índices de calidad de lec- 

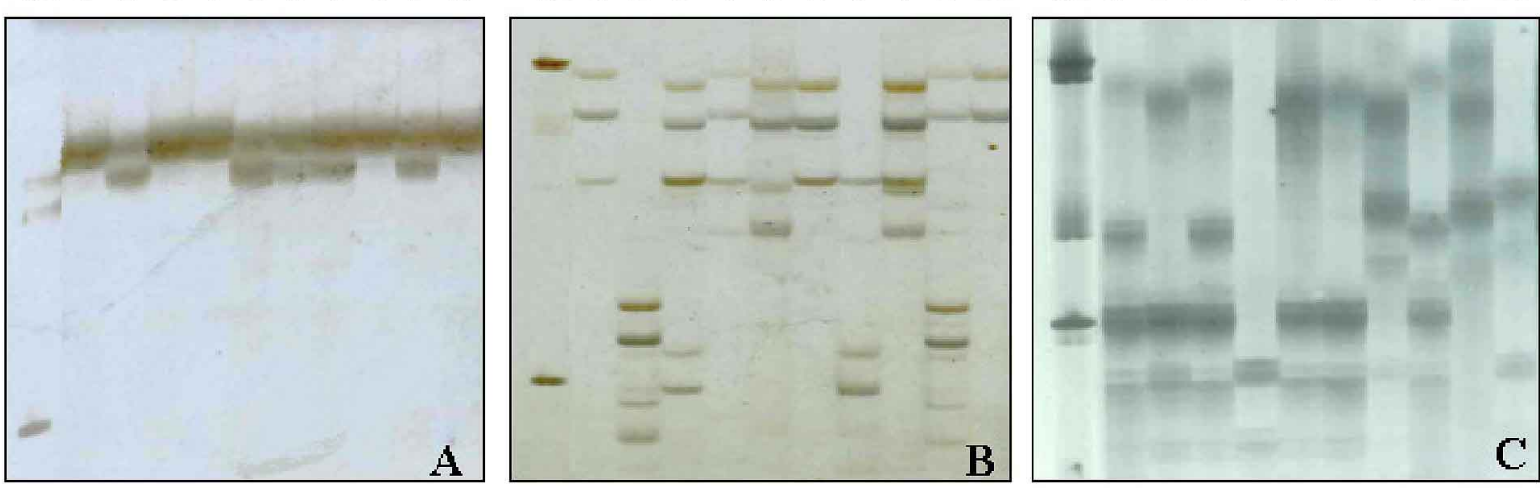

Figura 1. Perfiles de ADN para tres SSRs que presentan diferentes calidades de lectura de banda: (A) STM1053 con calidad de lectura de 1; (B) STM0031 con calidad de lectura de 2; y (C) STM1009 con calidad de lectura de 3. Figure 1. DNA profiles for three SSRs showing different band quality scoring: (A) STM1053 with scoring quality 1 ; (B) STM0031 with scoring quality 2; and (C) STM1009 with scoring quality 3.

tura de 1,2 y 3 , respectivamente. En este trabajo, la mayoría de los marcadores presentaron una buena calidad salvo los marcadores STM1106 (3), STM1009 (3), STM0031 (2) y STM1017 (2) los cuales, bajo las condiciones de laboratorio utilizadas, presentaron baja nitidez además de bandas tartamudas y presencia de sombras, lo que dificultó la lectura de alelos (Cuadro 1).

\section{Poder discriminatorio de los SSR}

Como se observa en el Cuadro 1, cada marcador fue evaluado según el valor del contenido de información polimórfica (PIC). Este parámetro es frecuentemente utilizado para medir la capacidad discriminatoria de marcadores SSRs; sin embargo, su valor puede variar para un mismo SSR, dependiendo de las características del germoplasma estudiado (Morales, 2002). El PIC calculado para un marcador puede variar entre 0 y 1 , indicando un mayor nivel de polimorfismo o variación cuando el valor es más cercano a 1. Paralelamente, la estimación del poder discriminatorio esperado $(\mathrm{Dj})$ de cada SSR, al igual que el valor de PIC se basa en las frecuencias alélicas y por lo tanto, corresponden a valores prácticamente idénticos (Tessier et al., 1999), lo cual se observó en los resultados obtenidos (Cuadro 1).

Los valores Dj para el total de marcadores (Cuadro 1) estuvieron dentro del rango de 0,92 y 0,43 , donde los siete marcadores de mayor poder discriminante fueron STM1009 $(0,92)$, STM0031 $(0,90)$, STM1020 $(0,86)$, STM3023 y STM2013 $(0,84)$, STM1008 y STM2022 $(0,82)$. En este grupo están incluidos los marcadores SSR multi-lociSTM1009, STM1020 y STM1008. En este caso, el valor $\mathrm{Dj}$ equivale a la información combinada de los alelos de sus respectivos loci. En general, los niveles de polimorfismo detectados para la mayoría de los marcadores fueron similares a los descritos por Ghislain et al. (2001a; b; 2004), quienes han evaluado cultivares de papa provenientes de India y Sud América, y una colección de cerca de mil accesiones de $S$. tuberosum, respectivamente. Esta última colección estaba constituida por ocho grupos taxonómicos diferentes de Stenotomum, Phureja, Ajanhuiri, Chaucha, Juzepczukii, Andigenum, Chilotanum, Curtilobum (Ghislain et al., 2004).

Por otro lado, al comparar los valores del poder de discriminación observado (Do) y sus respectivos valores esperados $(\mathrm{Dj})$ se aprecia que en la mayoría de los casos el valor observado es mayor al esperado (Figura 2). Probablemente esta diferencia tendería a disminuir a medida que se aumente el número de genotipos de papa en el análisis. Sin embargo, como se observa en la Figura 2, la tendencia la rompen los marcadores STM3023, STM2022, STM1053 y STM2030, donde Do resultó menor que Dj. Sólo un caso, STM1058, presentó valores idénticos para ambos parámetros. Esto indica que la capacidad discriminatoria de un marcador no sólo depende de las frecuencias alélicas, sino también de la forma en que están combinados los alelos en los genotipos de papa evaluados, pudiendo dos marcadores con igual valor de PIC y $\mathrm{Dj}$, presentar poderes discriminatorios observados (Do) muy diferentes. Los siete marcadores que presentaron un 


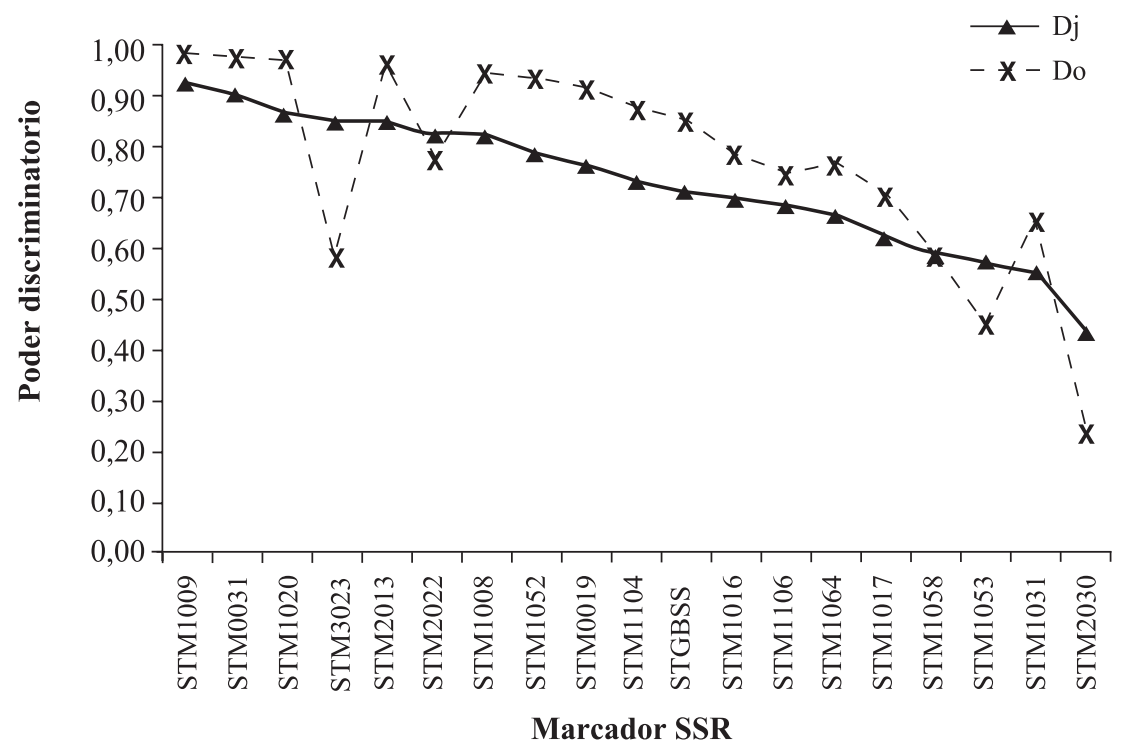

Figura 2. Comparación de poderes discriminatorios observado (Do) y esperado (Dj) de SSRs.

Figure 2. Comparison of observed (Do) and expected (Dj) SSRs discriminatory powers. SSR: simple sequence repeat.

mayor valor Do fueron STM1009 (0,98), STM1020 y STM0031 (0,97), STM2013 (0,96), STM1008 $(0,94)$, STM1052 $(0,93)$ y STM0019 $(0,91)$ (Cuadro 1$)$.

Respecto al número de combinaciones alélicas observadas (Ao), en todos los casos se presentaron valores inferiores al máximo de combinaciones posibles $(\mathrm{Aj})$ en individuos tetraploides. Para un marcador SSR de simple locus, sobre cinco alelos el número máximo de genotipos posibles sobrepasa el tamaño de la población analizada, por lo tanto en estos casos se esperan valores observados menores. Sin embargo, para un número de alelos $\leq 5$ se obtienen valores de $\mathrm{Aj}$ de $\leq 70$, pero las respectivas combinaciones observadas Ao fueron siempre menores. Todo esto indica que existen combinaciones alélicas que se repiten, posiblemente por la selección a que han sido sometidos estos materiales antes de convertirse en cultivares o germoplasma avanzado. Es destacable la situación del marcador STM3023, en el cual con siete alelos y 210 posibilidades de combinaciones alélicas diferentes sólo se encontraron cinco combinaciones (Cuadro 1).

\section{Combinaciones óptimas de SSRs para identifi- cación de genotipos}

En general todos los métodos de identificación confiables recomiendan incluir el mayor número posi- ble de parámetros descriptores, lo cual también es válido en el uso de marcadores moleculares. Sin embargo, para disminuir costos y aumentar la eficiencia total del análisis, es necesario determinar un número mínimo de marcadores para los cuales el riesgo de confundir germoplasma sea muy bajo (Narvaez et al., 2001).

Con el objetivo de definir un grupo de marcadores altamente informativos que permita diferenciar la totalidad del germoplasma utilizado, los marcadores incluidos en este análisis se ordenaron por valor de Do decreciente y se combinaron según se indica en el Cuadro 5. Para cada combinación de marcadores se determinó el número esperado y observado de pares de genotipos que pueden ser confundidos, Xk y Xo, respectivamente, para una población de tamaño $n=71$. El valor esperado Xk se hace igual a cero al utilizar cinco o más loci (STM1009/STM0031/STM3023/ STM2013/STM1020). Sin embargo, los valores observados Xo muestran que al utilizar tres o más loci (STM1009/STM0031/STM3023) es posible distinguir la totalidad del germoplasma (Cuadro 5). Lo mismo ocurre con la combinación STM1020/STM2013/STM1052, aunque estos marcadores presentaron menores valores de PIC, mostraron mayor facilidad de lectura si son comparados con los perfiles obtenidos con STM1009 y STM0031. 
Cuadro 5. Poder discriminatorio acumulado de las combinaciones de marcadores SSR más informativas. Table 5. Accumulative discriminatory power of the most informative SSR markers combinations.

\begin{tabular}{lccc}
\hline Combinaciones de marcadores SSRs & \multicolumn{2}{c}{$\mathbf{N}^{\mathbf{0}}$ de pares de genotipos no diferenciados } \\
\cline { 3 - 4 } \multicolumn{1}{l}{ Esperado (Xk) } & Observado (Xo) \\
\hline $\mathrm{A}+\mathrm{B}$ & 173,95 & 45 \\
$\mathrm{~A}+\mathrm{B}+\mathrm{C}$ & 97,41 & 4 \\
$\mathrm{~A}+\mathrm{B}+\mathrm{C}$ & 14,61 & 0 \\
$\mathrm{~A}+\mathrm{B}+\mathrm{C}+\mathrm{D}$ & 2,19 & 0 \\
$\mathrm{~A}+\mathrm{B}+\mathrm{C}+\mathrm{D}+\mathrm{E}$ & 0,32 & 0 \\
$\mathrm{~A}+\mathrm{B}+\mathrm{C}+\mathrm{D}+\mathrm{E}+\mathrm{F}$ & 0,03 & 0 \\
$\mathrm{~A}+\mathrm{B}+\mathrm{C}+\mathrm{D}+\mathrm{E}+\mathrm{F}+\mathrm{G}$ & 0,00 & 0 \\
$\mathrm{~A}+\mathrm{B}+\mathrm{C}+\mathrm{D}+\mathrm{E}+\mathrm{F}+\mathrm{G}+\mathrm{H}$ & 0,00 & 0 \\
$\mathrm{~A}+\mathrm{B}+\mathrm{C}+\mathrm{D}+\mathrm{E}+\mathrm{F}+\mathrm{G}+\mathrm{H}+\mathrm{I}$ & 0,00 & 0 \\
\hline
\end{tabular}

A: STM1009, B: STM0031, C: STM3023, D: STM2013, E: STM1020, F: STM3012/STPoAc58, G: STM2022,

H: STM1008 e I: STM1052. SSR: simple sequence repeat.

\section{Reducción de costos en el análisis de marcado- res SSR}

Dentro de los desafíos que deben enfrentar los marcadores moleculares para poder ser aplicados en forma rutinaria en identificación de germoplasma, se encuentra reducir los costos de análisis. Éstos pueden ser reducidos considerablemente optimizando métodos alternativos rápidos de extracción de ADN y utilizando PCR en condición de multiloci, múltiplex y pseudos-múltiplex.

Desde el punto de vista de la extracción de ADN, sin considerar la mano de obra, los costos pueden ser reducidos sobre un $90 \%$ utilizando procedimientos alternativos como el método de lisis alcalina descrito por Paris y Carter (2000). Aplicando este protocolo rápido, en el presente estudio, el ADN de una muestra pudo ser extraído en menos de 1 min y con un costo de $\$ 44$ (US $\$ 0.08$ ) por muestra. Es importante destacar que la calidad de este ADN como templado para reacciones PCR fue evaluado bajo las condiciones de trabajo del laboratorio de INIA-Remehue, obteniéndose resultados similares a los métodos convencionales, aunque los productos de SSR presentaron una menor intensidad (Sagredo et al., 2004). Además, estas muestras de ADN extraído con el método rápido se mostraron estables después de haber sido congeladas y descongeladas después de 3 meses. Sin embargo, un pequeño porcentaje de las muestras (aprox. 1\%) nunca generaron productos de amplificación, lo cual aparentemente se debe a la presencia de contaminantes que inhiben la reacción de PCR.

Otra posibilidad para aumentar la eficiencia del análisis con marcadores SSRs, es utilizar marcado- res multi-loci, los cuales con un solo par de partidores amplifican productos de ADN de más de una región del genoma (Cuadro 1). Los SSR multilocus utilizados correspondieron a STM0040 ( $C r$. III y VII), STM1008 ( $C r$. II, IV y IX), STM1050 ( $C r$. III, IV, VI y VII), STM1009 (Cr. VII y XI) y STM1020 (Cr. III y V) (Milbourne et al., 1998). No obstante, en este estudio sólo fue seleccionado STM1020 por el nivel de nitidez observado en los geles. En general, los SSRs multi-loci permitieron observar mayor número de alelos por genotipo, lo que resultó en un mayor poder discriminatorio observado (Cuadro 1). La gran limitación de este tipo de marcadores SSR multi-loci es su escaso número descrito en papa.

La alternativa de realizar una reacción de PCR simultánea para más de un SSR, llamado PCR-múltiplex, permite reducir los costos y tiempos a la mitad, tanto en la PCR como en la electroforesis. Sin embargo, debe existir compatibilidad de tamaño de productos PCR y de las temperaturas de apareamiento de partidores durante la PCR. En este caso, la mejor posibilidad correspondió a la combinación STPoAc58/STM3012, con la cual fue posible reconocer sólo un $18 \%$ del germoplasma estudiado (Cuadro 2).

El uso de pseudos-múltiplex, que consiste en mezclar los productos PCR de SSRs que presentan productos de diferente tamaño, es una alternativa bastante recurrida en los análisis de marcadores SSR (Ghislain et al., 2004). En este caso, la reducción de costos se produce sólo en la etapa de la electroforesis. Sin embargo, es la mejor posibilidad para utilizar las mejores combinaciones de marcadores 
SSR de mayor poder discriminatorio. En el presente análisis se logró reconocer un 78\% del germoplasma con la combinación STM2013/STM1052 y $42 \%$ con STM1016/STM1104/STGBSS (Cuadro 2 y Figura 3).

Dentro del grupo de 71 genotipos analizados, 15 corresponden a cultivares del Registro de Variedades descritas oficialmente por el Servicio Agrícola y Ganadero (SAG), de Chile, y corresponden a los cultivares Atlantic, Asterix, Cardinal, Desirée, Granola, Karu-INIA, Kennebec, OnaINIA, Oscar, Pukará-INIA, Ranger Russet, Romano, Rosara, Shepody y Yagana-INIA. Éstas se pueden distinguir totalmente con los partidores STM1020, STM2013 y STM1052 (Mathias et al., 2004). Recientemente, seis de los mejores SSRs que se caracterizaron en este estudio se evaluaron para identificación varietal en grupo de 80 geno- tipos que corresponden a todos los cultivares de papa de la Lista de Variedades Descritas Oficialmente del SAG del año 2004, pudiéndose diferenciar e identificar el $100 \%$ de las variedades analizadas (Manuscrito en preparación). Estos resultados y los obtenidos en otros laboratorios (Norero et al., 2003; Coombs et al., 2004) de identificación de cultivares mediante marcadores SSR, demuestran que ésta es una herramienta que puede impactar positivamente la comercialización de semilla certificada, tanto en los programas de certificación como para la protección de los derechos del obtentor.

\section{CONCLUSIONES}

Los resultados de la evaluación de marcadores microsatélites como herramienta de identificación de genotipos de papa, permitió seleccionar un grupo
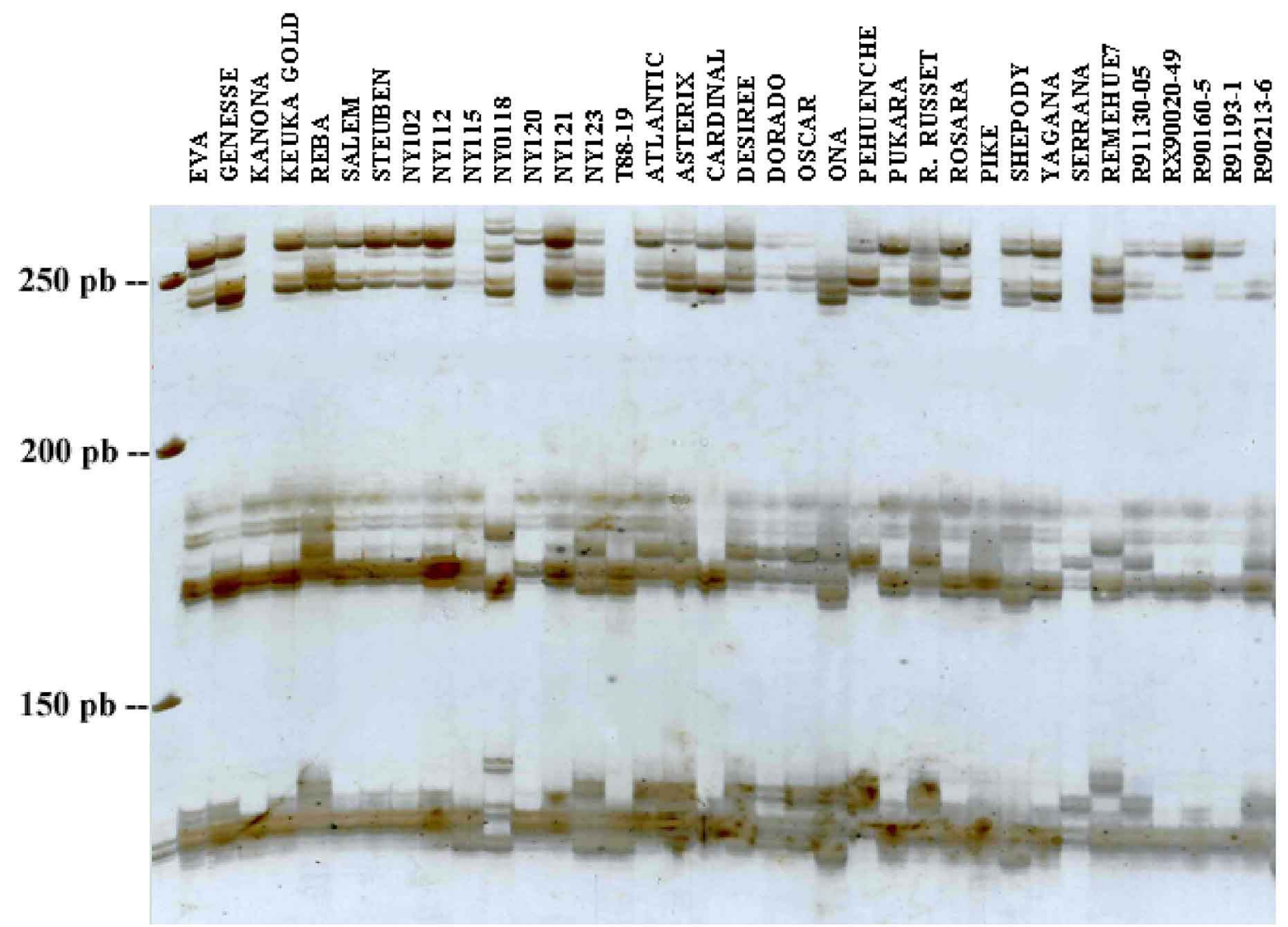

Figura 3. Perfiles de ADN para un grupo de variedades y materiales avanzados de papa utilizando SSRs en condición de pseudos múltiplex (STM1016/STM1104/STGBSS).

Figure 3. DNA profile for a group of potato varieties and advanced materials using pseudo multiplex SSRs (STM1016/STM1104/STGBSS). 
de marcadores SSR altamente informativos para facilitar la conservación y manejo del germoplasma de papa del programa de mejoramiento genético de papa de INIA-Remehue.

Se recomienda para identificación de genotipos de papa, el uso de los marcadores STM1020, STM2013 y STM1052, por su alta capacidad de discriminación, facilidad de lectura e interpretación y por la posibilidad de ser utilizados en condición de pseudos-múltiplex.

El desarrollo de protocolos que utilicen marcadores multi-loci, PCR-múltiplex y/o PCR pseudosmúltiplex, junto con la aplicación de métodos de extracción de ADN más simples y rápidos, aumentan la factibilidad de uso de este tipo de tecnología para la conservación y manejo de germoplasma de papa, ya que disminuyen ostensiblemente el tiempo y costo de los análisis.

En comparación con los marcadores morfo-agronómicos, los marcadores SSR presentan grandes ventajas para la identificación de cultivares de papa, y su utilización podría fortalecer los sistemas de producción y comercialización de semilla de papa.

\section{RECONOCIMIENTO}

Este trabajo fue desarrollado gracias el apoyo financiero de la Fundación para la Innovación Agraria, Proyecto FIA-BIOT-01-A-015.

\section{LITERATURA CITADA}

Anderson, J.A., G.A. Churchill, J.E. Autroque, S.D. Tanksley, and M.E. Swells. 1993. Optimizing selection for plant linkage map. Genome 36:181-186.

Ashkenazi, V., E. Chani, U. Lavi, D. Levy, J. Hillel, and E. Veilleux. 2001. Development of microsatellite markers in potato and their use in phylogenetic and fingerprinting analyses. Genome 44:50-62.

Becerra, V.M., A. Paredes, A. Romero, y L. Lavin. 2001. Diversidad bioquímica y molecular en frutillas chilenas (Fragaria chiloensis L. Duch.) y su implicancia en el mejoramiento de la especie. Agric. Téc. (Chile) 61:413-428.

CIP. 2004. Taller de análisis SSR en papa y desarrollo de bases de datos. 25-29 de octubre 2004. Lima, Perú. Annual Report 2004. Centro Internacional de la Papa (CIP), Lima, Perú.

Coombs, J.J, L.M. Frank, and D. S. Douches. 2004. An applied fingerprinting system for cultivated potato using simple sequence repeats. Am. J. Potato Res. 81:243-250.

Fulton, T.M., J. Chunwongse, and S.D. Tanksley. 1995. Microprep protocol for extraction of DNA from tomato and other herbaceous plants. Plant Mol. Biol. Report 13:207-209.

Ghislain, M., F. Rodriguez, J. Núñez, P. Naik, Z. Huamán, and M. Bonierbale. 2001a. Comparison of genetic diversity of potato varieties from India and South America. Poster presented at Plant and Animal Genome, San Diego, California, USA. January 13-17. Available at http://www.cipotato.org Accessed 27 September 2004.
Ghislain, M., F. Rodriguez, F. Villamon, J. Núñez, R. Waugh, and M. Bonierbale. 2001b. Establishment of microsatellite assays for potato genetic identification. p. 167-174. In International Potato Center (CIP), Scientist and farmer: Partners in research for the 21st century. Program Report, 1999-2000. Lima, Peru.

Ghislain, M., D.M. Spooner, F. Rodriguez, F. Villamón, J. Núñez, C. Vásquez, et al. 2004. Selection of highly informative and user-friendly microsatellites (SSRs) for genotyping of cultivated potato. Theor. Appl. Genet. 108:881-890.

Hinrichsen, P., C. Amigo, R. Alvarado, y C. Muñoz. 1996. Identificación de variedades chilenas de arroz (Oryza sativa L.). Evaluación del uso de perfiles proteicos y de fragmentos polimórficos de ADN amplificados al azar (RAPD). Agric. Téc. (Chile) 56:1-10.

Kawchuck, L.M., D.R. Lynch, J. Thomas, B. Penner, D. Sillito, and F. Kulcsar. 1996. Characterization of Solanum tuberosum simple sequence repeats and application to potato cultivar identification. Am. Potato J. 73:325-335.

Kruglyak, S., R.T. Durrett, M.D. Schug, and C.F. Aquadro. 1998. Equilibrium distributions of microsatellite repeat length resulting from a balance between slippage events and points mutations. Proc. Natl. Acad. Sci. USA 95:10774-10778.

Liu, B.H. 1998. Statistical genomics: linkage, mapping and QTL analysis. 611 p. CRC Press, Boca Raton, Florida, USA. 
Mathias, M., B. Sagredo, y J. Kalazich. 2004. Identificación de cultivares de papa a través de marcadores SSR en Chile. p. 92. XXI Congreso de la Asociación Latinoamericana de la Papa (ALAP), Valdivia, Chile. 712 marzo de 2004. Asociación Latinoamericana de la Papa, Valdivia, Chile.

Milbourne, D., R.C. Meyer, A.J. Collins, L.D. Ramsay, C. Gebhardt, and R. Waugh. 1998. Isolation, characterization and mapping of simple sequence repeats loci in potato. Molecular Genetics 259:233-245.

Morales, L. 2002. Caracterización genotípica de plantas de maíz (Zea mays) utilizando secuencias microsatélites distribuidas uniformemente sobre el genoma. Tesis Licenciado en Ciencias Biológicas. 34 p. Universidad de Belgrano, Facultad de Ciencias Exactas y Naturales, Buenos Aires, Argentina.

Narvaez, C. 1998. Uso de marcadores moleculares para la identificación de variedades de vid (RAPD, AFLP, SSR). 59 p. Tesis Bioquímico. Universidad de Chile, Facultad de Ciencias Químicas y Farmacéuticas, Santiago, Chile.

Narvaez, C., H. Castro, J. Valenzuela, y P. Hinrichsen. 2001. Patrones genéticos de los cultivares de vides de vinificación más comúnmente usados en Chile basados en marcadores de microsatélites. Agric. Téc. (Chile) 61:249-261.

Norero, N., M. Huarte, y S. Feingold. 2003. Potente herramienta para la identificación de cultivares de papa. Presentado como poster al V Simposio de Recursos Genéticos para América Latina y el Caribe, Mar del Plata, 10 a 14 noviembre 2003. Disponible en http:// www.inta.gov.ar Leído el 15 de octubre de 2004.

Ortiz, J.M., I. Aguinagalde, y J.P. Martín. 2000. Identificación varietal. p. 515-560. In F. Nuez y J. M. Carrillo (eds.). Los marcadores genéticos en la mejora vegetal. Editorial de la UVP, Valencia, España.

Paredes, M., V. Becerra, R. Avilés, C. Rojo, C. Balocchi, J. Zapata, y F. Dropelmann. 2004. Desarrollo e implementación de herramientas moleculares para la caracterización de material genético forestal, proyecto FDI-CORFO, 2001-2004. Disponible en http:// www.inia.cl Leído el 20 de julio de 2004.

Paris, M., and M. Carter. 2000. Cereal DNA: A rapid HighThroughput extraction method for marker assisted selection. Plant Mol. Biol. Reptr. 18:357-360.

Perazzo, G., A. Panta, F. Rodriguez, R. Gomez, J. Toledo, Z. Huaman, et al. 2001. Clonal true to type verification of potato accessions retrieved from in vitro conservation and cryopreservation. p. 175-183. In Scientist and farmer: Partners in research for the 21st century. Program Report, 1999-2000. International Potato Center (CIP), Lima (Peru).
Promega. 2004. Technical manual: Silver sequence DNA sequencing system. Instructions for use of products Q4130 and Q4131. Available at http://www. promega.com Accessed 12 January 2004.

Provan, J., W. Powell, and R. Waugh. 1996. Microsatellite analysis of relationships within cultivated potato (Solanum tuberosum). Theor. Appl. Genet. 92:10781084.

Riegel, R., R. Pérez, M. Mathias, y A. Contreras. 2004. Diversidad y relaciones genéticas en papas nativas silvestres y cultivadas en Chile. p. 54. XXI Congreso de la Asociación Latinoamericana de la Papa (ALAP), Valdivia, Chile. 7-12 de marzo 2004. Asociación Latinoamericana de la Papa, Valdivia, Chile.

SAG. 2005. Servicio Agrícola y Ganadero: certificación de semillas agrícolas. Disponible en http://www.sag.cl Leído el 20 de abril de 2004.

Sagredo, B., M. Mathias, y J. Kalazich. 2004. Identificación varietal de papa mediante marcadores SSR en múltiplex y pseudos-múltiplex. p. $161.55^{\circ}$ Congreso Agronómico de Chile y $5^{\circ}$ Congreso de la Sociedad Chilena de Fruticultura, Valdivia, Chile. 19-22 de noviembre 2004. Sociedad Agronómica de Chile y Sociedad Chilena de Fruticultura, Valdivia, Chile.

Sambrook, J., and D. Russell. 2001. Molecular cloning: A laboratory manual. $3^{\text {rd }}$ ed. Cold Spring Harbor Laboratory Press, New York, USA.

Schneider, K., and D. Douches. 1997. Assessment of PCRbased sequence repeats to fingerprint North American potato cultivars. Am. Potato J. 74:149-160.

Sneath, P.H.A., and R.R. Sokal. 1973. Numerical taxonomy. 573 p. Freeman, San Francisco, California, USA.

Tessier, C., J. David, P. This, J.M. Boursiquot, and A. Charrier. 1999. Optimization of the choice of molecular markers for varietal identification in Vitis vinifera L. Theor. Appl. Genet. 98:171-177.

UPOV. 2001. International Union for the Protection of New Varieties of Plants-UPOV. Available at http:// www.upov.int/en/about/upov_system.htm Accessed 6 May 2004.

Veilleux., R.E., L.Y. Shen, and M.M. Paz. 1995. Analysis of the genetic composition of anther derived potato by randomly amplified polymorphic DNA and simple sequence repeats. Genome 38:1153-1162.

Zerené, M., D. Granger, D. Prehn, y P. Hinrichsen. 2000. Secuencias de microsatélites asociadas a genes de proteínas de reserva en variedades chilenas de trigo harinero: descripción y posible uso como marcadores moleculares. Agric. Téc. (Chile) 60:14-31. 